January, 1999

TAC-1999-001

\title{
Neutrino Degeneracy and Chemically Inhomogeneous Universe ${ }^{1}$
}

\author{
A.D. Dolgov \\ Teoretisk Astrofysik Center \\ Juliane Maries Vej 30, DK-2100, Copenhagen, Denmark Đ
}

\begin{abstract}
A possibility that the universe may be strongly inhomogeneous chemically, while very smooth energetically, is considered. A possible mechanism which could lead to such a picture is a large and inhomogeneous lepton asymmetry. The model may explain possibly observed variation of primordial deuterium by an order of magnitude and predicts helium-rich and possibly helium-poor regions where the helium mass fraction differs from the observed $25 \%$ by a factor of 2 in either direction. Helium variation can be observed by a different dumping rate of $\mathrm{CMB}$ angular spectrum at small angles at different patches of the sky.
\end{abstract}

It is usually assumed that the universe is well described by a homogeneous and isotropic matter distribution. Of course at the present day the matter is quite clumpy and the mass (or energy) density contrast is large at galactic and even at cluster scales. Averaged over larger scales the matter distribution is probably very smooth. The observed clumpiness evolved from initially small density fluctuations due to gravitational instability. The smoothness of the young universe is strongly confirmed by very small angular fluctuations of the temperature of the cosmic microwave (CMB) radiation. Though the energetic homogeneity of the universe is well verified up to red shift $z=10^{3}$, corresponding to the last scattering of CMB, its chemical homogeneity remains an assumption, maybe quite natural, but still an assumption. In what

\footnotetext{
${ }^{1}$ To be published in "Particle Physics and the Early Universe (COSMO-98)", ed. by David O. Caldwell American Institute of Physics

${ }^{2}$ Also: ITEP, Bol. Cheremushkinskaya 25, Moscow 113259, Russia.
} 
follows I will consider the question if a big chemical inhomogeneity at cosmologically large scales could be compatible with the observed smooth mass or energy distribution in the universe. We discuss a possible mechanism which may give rise to a large variation of element abundances in the early universe, preserving cosmological homogeneity, $\delta \rho / \rho \ll 1$, and possible manifestations of this variation of abundances. To a large extent my talk is based on our paper with B. Pagel [1].

The starting point of this investigation from the observational side was the reported measurements [2] - [8] of the deuterium abundance in Lyman-limit absorption line systems with red-shifts $0.48<z<3.5$ on the line of sight to quasars. Surprisingly some groups have claimed a very high value for the deuterium-to-hydrogen ratio, $\mathrm{D} / \mathrm{H} \approx(\mathrm{a}$ few $) \times 10^{-4}$. Though these results are subject to different kinds of criticism [4, 9, 10], they put forward a very important question: what is known about element abundances at very large distances?

¿From theoretical point of view it is an interesting challenge to find if there exist (not too unnatural) cosmological scenarios consistent with the observed smoothness of the universe but predicting large abundance variations. An example of such mechanism was proposed in ref. [11] (see also [12]), where a model of leptogenesis was considered which, first, gave a large lepton asymmetry, which could be close to unity, and, second, this asymmetry might strongly change on astronomically large scales, $l_{L}$. The magnitude of the latter depends on the unknown parameters of the model and can easily be in the mega-giga parsec range. The model is based on the AffleckDine [13] scenario of baryogenesis but in contrast to the original one it gives rise to a large (and varying) lepton asymmetry and to a small baryonic one. There are two more models [14]-116] in the recent literature, where a large lepton asymmetry together with a small baryonic one is advocated, though without any significant spatial variation.

If we assume that the variation of deuterium abundance by approximately an 
order of magnitude is indeed real, then the characteristic scale $l_{L}$ should be smaller than a gigaparsec. The lower bound on this scale may be much smaller. It can in principle be determined by measurements of the abundances of light elements at large distances in our neighborhood, say, $z \geq 0.05$.

A variation of deuterium abundance may be also explained by a variation of the cosmic baryon-to-photon ratio. This possibility was explored in refs. [17, 18]. The isocurvature fluctuations on large scales, $l>100 \mathrm{Mpc}$, which are necessary to create the observed variation of deuterium, are excluded [18] by the smallness of angular fluctuations of the cosmic microwave background radiation (CMB). Variations of baryonic number density on much smaller scales, $M \sim 10^{5} M_{\odot}$, are not in conflict with the observed smoothness of CMB and in principle can explain the data subject to a potential conflict with the primordial ${ }^{7} \mathrm{Li}$ abundance [17].

Exactly the same criticism of creating too large fluctuations in CMB temperature is applicable to a simple version of the model with a varying lepton asymmetry. One can check that the necessary value of the chemical potential of electron neutrinos $\xi_{\nu_{e}}$ should be close to -1 to explain the possibly observed variation of deuterium by roughly an order of magnitude. Such a change in $\xi_{\nu_{e}}$ with respect to zero value, assumed in our part of the world, would induce a variation in total energy density during the RD stage at a per cent level, which is excluded by the smoothness of CMB. However, this objection can be avoided if there is a conspiracy between different leptonic chemical potentials such that in different spatial regions they have the same values but with interchange of electronic, muonic and/or tauonic chemical potentials. Since the abundances of light elements are much more sensitive to the magnitude of the electron neutrino chemical potential than to those of muon and tauon neutrinos, the variation of $\xi_{\nu_{e}}$ (accompanied by corresponding variations of $\xi_{\nu_{\mu}}$ and $\xi_{\nu_{\tau}}$ ) would lead to a strong variation in the abundance of deuterium and other light elements. The equality of, say, $\xi_{\nu_{e}}$ at one space point to $\xi_{\nu_{\mu}}$ at another point looks like a very 
artificial fine-tuning, but this may be rather naturally realized due the lepton flavor symmetry, $e \leftrightarrow \mu \leftrightarrow \tau$.

If lepton asymmetry changes at large distances, then not only deuterium but also ${ }^{4} \mathrm{He}$ would not remain constant in space. Playing with the nucleosynthesis code 19 one can check that in the deuterium rich regions the mass fraction of helium could be larger than 50\% (twice larger than in our neighborhood). There may also exist the so called mirror regions with a positive and large chemical potential of electronic neutrinos. In such regions abundances of both deuterium and helium would be much smaller that those observed nearby. For more detail see ref. [1]. Surprisingly nothing is known about helium abundance at large distances. All accurate measurements of ${ }^{4} \mathrm{He}$ based on emission lines known to us were done at most at $z=0.045$ corresponding to a distance of $140 h^{-1} \mathrm{Mpc}$ [20], whereas helium line and continuum absorption measurements made at high redshifts give the abundance merely within "a factor of a few" owing to uncertain ionization corrections [21]. In the regions with a large fraction of ${ }^{4} \mathrm{He}$ one would expect bluer stars with a shorter life-time, though the structure formation there may be inhibited due to a less efficient cooling. In the helium poor regions the effects may be opposite. As was noted by A. Kusenko at this Conference, a study of supernovae may help to put limits on abundance variation. This problem definitely deserves further and more detailed investigation.

Lepton conspiracy, mentioned above, would diminish energy density fluctuations in first approximation. However there are some more subtle effects which could be either dangerous for the model or observable in CMB. The first one is related to the binding energy of ${ }^{4} \mathrm{He}\left(7 \mathrm{MeV}\right.$ per nucleon). Since the mass fraction of ${ }^{4} \mathrm{He}$ may change by a factor of 2 in deuterium- (and helium-) rich regions (from $25 \%$ to more than 50\%), this means that the variation in baryonic energy density may be as large as $2 \cdot 10^{-3}$. Rescaling the estimates of ref. [18] one can find [1] for the fluctuations of the CMB temperature: $\delta T / T \approx 10^{-5}\left(R_{\text {hor }} / 10 \lambda\right)$, where $\lambda$ is the wavelength of the 
fluctuation and $R_{h o r}$ is the present day horizon size. The restriction on the amplitude of temperature fluctuations would be satisfied if $\lambda>200-300 \mathrm{Mpc} / h_{100}\left(h_{100}=\right.$ $H / 100 \mathrm{~km} / \mathrm{sec} / \mathrm{Mpc}$ ). Surprisingly direct astrophysical effects of such big fluctuations of the helium mass fraction at distances above $100 \mathrm{Mpc}$ cannot be observed presently, at least the evident simple ones.

Another effect which would induce energy inhomogeneities, is the heating of neutrinos by $e^{+} e^{-}$-annihilation at $T \leq 1 \mathrm{MeV}$, when neutrinos practically decoupled from plasma, and the corresponding cooling of photons. (For the most recent and precise calculations of this cooling see ref. [23]). The efficiency of the cooling depends upon the chemical potential of neutrinos and would create fluctuations in CMB temperature at the level of $2 \cdot 10^{-5}$ [1].

A variation of mass fraction of primordial ${ }^{4} \mathrm{He}$ could be observed in the future high precision measurements of CMB anisotropies at small angular scales [22]. There are two possible effects, first, a slight difference in recombination temperature which logarithmically depends on hydrogen-to-photon ratio, and second, a strong suppression of high multipoles with an increase of $R_{p}$. The latter is related to the earlier helium recombination with respect to hydrogen and correspondingly to a smaller number of free electrons at the moment of hydrogen recombination. This in turn results in an increase of the mean free path of photons in the primeval plasma and in a stronger Silk damping [24]. The position and the magnitude of the first acoustic peak remains practically unchanged [22].

This effect seems to be very promising for obtaining a bound on or an observation of a possible variation of primordial helium mass fraction. If this is the case then the amplitude of high multipoles at different directions on the sky would be quite different. The impact of the possible variation of primordial abundances on the angular spectrum of CMB anisotropy at low $l$ is more model dependent. It may have a peak corresponding to the characteristic scale $R>200-300 \mathrm{Mpc}$ or a plateau, 
which would mimic the effect of the hot dark matter.

Acknowledgments. The work of A.D. was supported by Danmarks Grundforskningsfond through its funding of the Theoretical Astrophysical Center.

\section{References}

[1] A.D. Dolgov and B.E.J. Pagel, New Astronomy (to be published); astroph/9711202.

[2] D. Tytler, X-M. Fan, and S. Burles, Nature 381 (1996) 207.

[3] M. Rugers and C.J. Hogan, Astrophys. J. 469, L1 (1996).

[4] D. Tytler, S. Burles, and D. Kirkman, astro-ph/9612121.

[5] A. Songalia, E.J. Wampler, and L.L. Cowie, Nature 385, 137 (1997).

[6] S. Burles and D. Tytler, astro-ph/9712109; Astrophys. J. (in press).

[7] J.K. Webb, R.F. Carswell, K.M.Lanzetta, et al. Nature, 388, 250 (1997).

[8] D. Tytler, S. Burles, L. Lu, et al, astro-ph/9810217.

[9] S.A. Levshakov, W.H. Kegel, and F. Takahara, astro-ph/9801108, submitted to A\&A Letters (1998); astro-ph/9802110, submitted to Astrophys. J. Letters. (1998).

[10] S.A. Levshakov, D. Tytler, and S. Burles, astro-ph/9812114.

[11] A.D.Dolgov and D.K.Kirilova, J.Moscow Phys.Soc., 1, 217 (1991).

[12] A.D.Dolgov, Phys.Repts. 222, No. 6 (1992).

[13] I. Affleck and M. Dine, Nucl. Phys. B249, 361 (1985). 
[14] R. Foot, M.J. Thompson, and R.R. Volkas, Phys. Rev. D 53, 5349 (1996);

[15] R. Foot and R.R. Volkas, Phys. Rev. D 55, 5147 (1997).

[16] A. Casas, W.Y. Cheng, and G. Gelmini, hep-ph/9709289.

[17] K. Jedamzik and G. Fuller,Astrophys. J. 452, 33 (1995)

[18] C.J. Copi, K.A. Olive, and D.N. Schramm, astro-ph/9606156.

[19] L. Kawano, Fermilab-Pub-92/04-A (1992).

[20] Y.I. Izotov, T.X. Thuan, and V.A. Lipovetsky, Astrophys. J. Suppl. 108, 1 (1997)

[21] C.J. Hogan, S.F. Anderson, and M.H. Rugers, Astr. J. 113, 1495 (1997).

[22] W. Hu, D. Scott, N. Sugiyama, and M. White, Phys. Rev. D 52, 5498 (1995).

[23] A.D. Dolgov, S.H. Hansen, and D.V. Semikoz, Nucl. Phys. B 524, 621 (1998).

[24] J. Silk, Astrophys. J., 151, 459 (1968). 